\title{
Relevance of EGFR Between Serum VEGF and MMP-9 in Primary Hepatocellular Carcinoma Patients with Transarterial Chemoembolization
}

This article was published in the following Dove Press journal: OncoTargets and Therapy

\author{
Shengya Cao' \\ Shuo Zhu ${ }^{2}$ \\ Wei Yin' \\ Heng $X u^{3}$ \\ Jianzhong $\mathrm{Wu}^{4}$ \\ Qiang Wang ${ }^{1,5}$ \\ 'Department of Clinical laboratory, \\ Xuzhou Cancer Hospital, Xuzhou, Jiangsu \\ 22 I005, People's Republic of China; \\ ${ }^{2}$ Department of Hepatobiliary Surgery, \\ Xuzhou Cancer Hospital, Xuzhou, Jiangsu \\ 22 1005, People's Republic of China; \\ ${ }^{3}$ Laboratory of Pharmaceutical \\ Chemistry, Jiangsu Province Institute of \\ Materia Medica, Nanjing Tech University, \\ Nanjing, Jiangsu 210000, People's \\ Republic of China; ${ }^{4}$ Research Center for \\ Clinical Oncology, Jiangsu Cancer \\ Hospital and Jiangsu Institute of Cancer \\ Research and Nanjing Medical University \\ Affiliated Cancer Hospital, Nanjing, \\ Jiangsu 210000, People's Republic of \\ China; ${ }^{5}$ Department of Radiotherapy, \\ Xuzhou Cancer Hospital, Xuzhou, Jiangsu \\ 221005, People's Republic of China
}

Correspondence: Jianzhong Wu Research Center for Clinical Oncology, Jiangsu Cancer Hospital and jiangsu Institute of Cancer Research and Nanjing Medical University Affiliated Cancer Hospital, No. 7 Building, 42 BaiZiTing Road, Nanjing, Jiangsu 210000, People's Republic of China

Email wujzh@jszlyy.com.cn

Qiang Wang

Department of Radiotherapy, Xuzhou Cancer Hospital, No. I3I, Huancheng

Road, Xuzhou, Jiangsu 221005, People's

Republic of China

Email doctorwang6I8@I26.com
Objective: The aim of this study was to estimate the relevance of the epidermal growth factor receptor (EGFR) between serum vascular endothelial growth factor (VEGF) and matrix metalloproteinase 9 (MMP-9) in primary hepatocellular carcinoma (HCC) patients with transarterial chemoembolization (TACE).

Methods: The pre-treatment and post-treatment concentrations of the serum VEGF and MMP-9 were detected with Luminex assay in 80 EGFR-negative patients and 59 EGFRpositive patients who received TACE therapy with different chemotherapeutic drugs.

Results: The serum concentration of MMP-9 in the EGFR-positive patients with primary HCC was significantly higher than that in the EGFR-negative patients $(P<0.05)$. In EGFRpositive patients with primary HCC, differences in stage, metastasis, and differentiation were significant $(P<0.05)$. Serum VEGF level significantly decreased at the second course of treatment in the EGFR-negative patients from the P group $(P<0.05)$, while serum MMP-9 level significantly decreased at the second course of treatment in the EGFR-negative patients from the E group $(P<0.05)$. Serum VEGF level in the EGFR-positive patients among three groups slightly decreased at the first, second and third courses of treatments; however, the differences were not significant $(P>0.05)$. Serum MMP-9 level in the EGFR-positive patients among three groups showed mild decrease at the first and second courses of treatments; however, the decreases at the third course of treatment were significant $(P<$ $0.05)$.

Conclusion: Serum VEGF and MMP-9 are potential biomarkers for the treatment monitoring of EGFR-positive and -negative patients after TACE therapy with different chemotherapeutic drugs.

Keywords: hepatocellular carcinoma, transarterial chemoembolization, EGFR, VEGF, MMP-9

\section{Introduction}

Liver cancer (LC) ranks as the fifth most common cancer and second leading cause of cancer-related death in men and the seventh most frequent cancer and sixth leading cause of death in women. ${ }^{1}$ Approximately 782,500 new cases and 745,500 deaths due to LC were reported worldwide in 2012. Half of the new cases of LC and related deaths worldwide occurred in China. ${ }^{2}$ Hepatocellular carcinoma (HCC) is the most common primary malignancy of liver. Across all countries, the 5-year overall survival (OS) is only $3 \%-5 \%$ in patients with HCC. ${ }^{3}$ Sorafenib has a median OS benefit in the range of 2.3-2.8 months. ${ }^{4}$ Unfortunately, the median 
OS for advanced HCC is less than 1 year. Molecular profiling is gaining attention in cancer diagnosis and treatment. EGFR (14\% of all patients) amplifications were identified by next-generation sequencing in advanced HCC. ${ }^{5}$ Epidermal growth factor receptor (EGFR) immunoexpression was determined in tissues from patients with HCC, with a mean value of $39.58 \%$, by immunohistochemical (IHC) staining. ${ }^{6}$ EGFR immunoexpression is essential for the efficacy and prognosis of patients with HCC to pay attention to the immunoexpression of EGFR in the progress of the disease.

EGFR is a transmembrane receptor that transforms growth factor $\alpha$ through EGF, which is one of its ligands. ${ }^{7}$ All the ligands of EGFR are synthesized as transmembrane precursors and cleaved by enzymes with metalloprotease activity. ${ }^{8}$ EGFR can regulate cell growth and division by activating the signal transduction pathways of cells and causes tumor cell proliferation and tumor neovascularization., ${ }^{9,10}$ Matrix metalloproteinase 9 (MMP-9) accelerates tumor metastasis by promoting neovascularization and lymphangiogenesis. ${ }^{11}$ Vascular endothelial growth factor (VEGF) is an important and effective factor that stimulates vascularization and participates in tumor invasion and metastasis. ${ }^{12}$ EGFR is overexpressed in the liver tissues of patients with HCC. ${ }^{13}$ Therefore, the relevance of EGFR between serum VEGF and MMP-9 in HCC should be evaluated.

In this study, the concentrations of serum VEGF and MMP-9 were measured in patients with primary HCC and healthy individuals. Meanwhile, the data of the EGFRpositive and -negative patients and their clinicopathological variables were investigated. The relevance of EGFR between serum VEGF and MMP-9 was estimated in primary HCC with transarterial chemoembolization (TACE).

\section{Materials and Methods}

\section{Patient Population}

A total 139 patients with histopathologically confirmed primary HCC were enrolled from Jiangsu Cancer Hospital (Nanjing, China) between January 2012 and July 2019 and from Xuzhou Cancer Hospital (Xuzhou, China) between June 2017 and May 2019. The patients were included in either the pre-treatment or post-treatment groups according to the courses of TACE therapy. The interval of every TACE therapy was 2-4 months. Initially, the patients were pathologically diagnosed with HCC and had not received any prior treatment in the pre-treatment group. Then, the patients received TACE therapy with different chemotherapeutic drugs and were divided into three groups according to basic chemotherapeutic drug. In the E group, 53 patients treated with adriamycin and its isomers (eg, epirubicin or adriamycin). In the $\mathrm{P}$ group, 44 patients treated with platinum (eg, oxaliplatin, nedaplatin, or lobaplatin). In the $\mathrm{O}$ group, 42 patients treated with other drugs (eg, 5-fluorouridine, recombinant endostatin). The characteristics of patients are shown in Table 1. The mean age of the patients was 57.0 years (SD, 11.0 years). A total of 183 healthy individuals (without any abnormalities) were enrolled, with a mean age of 48.5 years (SD, 13.8 years). Hepatitis B virus surface antigen (HBsAg) was determined by colloidal gold diagnostic reagent (ACON Laboratories, Zhejiang, China). Tumor recurrence was summarized as follows: After a period of treatment, the tumor could not be detected in the patient's body, and then the tumor returned by imaging diagnosis. The patients were staged according to the latest TNM staging issued in 2009 by the International Union Against Cancer. EGFR expression was detected in tissue slices from these patients by IHC staining with an antibody against EGFR (Monoclonal Mouse, Anti-Human, Maixin, Fuzhou, China).

\section{Serum Samples}

The patients were enrolled using a research protocol approved by the Biomedical Research Ethics Committee of Jiangsu Cancer Hospital and Xuzhou Cancer Hospital. This study was conducted in accordance with the Declaration of Helsinki. Each participant signed a written

Table I Clinical Features of Patients with HCC and the Controls

\begin{tabular}{|c|l|l|}
\hline Variables & Patients & Controls \\
\hline $\begin{array}{c}\text { Age (year) } \\
\text { Mean (SD) }\end{array}$ & $57.0 \pm 11.0$ & \\
$<50$ & $25.9 \%(36 / 139)$ & $48.5 \pm 13.8$ \\
$50-70$ & $64.7 \%(90 / 139)$ & $57.4 \%(105 / 183)$ \\
$>70$ & $9.4 \%(13 / 139)$ & $35.5 \%(65 / 183)$ \\
\hline $\begin{array}{l}\text { Sex } \\
\text { Male }\end{array}$ & & $7.1 \%(13 / 183)$ \\
Female & $81.3 \%(1 / 3 / 139)$ & $77.6 \%(142 / 183)$ \\
\hline HBsAg & $18.7 \%(26 / 139)$ & $22.4 \%(41 / 183)$ \\
Positive & & \\
Negative & $56.8 \%(79 / 139)$ & - \\
\hline EGRF & $43.2 \%(60 / 139)$ & - \\
Positive & & \\
Negative & $42.4 \%(59 / 139)$ & - \\
\hline Recurrence & $57.6 \%(80 / 139)$ & - \\
Yes & & - \\
No & $41.7 \%(58 / 139)$ & - \\
\hline
\end{tabular}


informed consent. Pre-treatment blood samples were collected. Blood samples from patients after TACE therapy with different chemotherapeutic drugs were collected at three different intervals (ie, first, second, and third administration of post-treatment). Before each course of TACE therapy, samples were collected from patients for VEGF and MMP-9 detection as treatment monitoring of last course. Samples were centrifuged at $4{ }^{\circ} \mathrm{C}$ for $10 \mathrm{~min}$, and stored at $-80^{\circ} \mathrm{C}$ until use.

\section{Detection of Serum VEGF and MMP-9 by Luminex Assays}

The assay was performed using a Luminex multiplex technique. FLEXMAP 3D instruments and software were supplied by Luminex Corporation (Austin, TX, USA). Luminex kits for human cytokine/chemokine panels (ie, VEGF and MMP-9) were acquired from Millipore (CAT nos. MPXHCYTO-60K-01 and HMMP2MAG-55K-01; Millipore Inc Billerica, MA, USA) and used according to the manufacturer's instructions. The preparation processes of the samples and parameters in FLEXMAP 3D were similar to those reported in a previous work. ${ }^{14}$ The serum VEGF and MMP-9 concentrations were calculated according to the standard curve using a known concentration.

\section{Statistical Analysis}

All data were analyzed by SPSS 19.0. A $P$ value of $<0.05$ was considered statistically significant. Serum VEGF and MMP-9 concentrations were presented as mean \pm SD. The differences between two groups were examined, and either a $t$-test or Mann-Whitney test was used in comparing the median values between the patient groups. Comparisons among multiple groups were performed using one-way ANOVA, followed by pair-wise comparisons using Bonferroni posthoc testing.

\section{Results}

\section{Serum VEGF and MMP-9 Levels in EGFR-Positive and -Negative Patients with Primary HCC}

Serum VEGF and MMP-9 were measured in 183 healthy individuals and 139 patients with primary $\mathrm{HCC}$, including 80 EGFR-positive patients and 59 EGFR-negative patients. The serum levels of VEGF were 145.2, 167.1, and $50.6 \mathrm{pg} / \mathrm{mL}$ in EGFR-negative patients, EGFR-positive patients, and controls, respectively (Figure 1A). Meanwhile, serum MMP-9 concentrations were $327.1,857.1$, and $1010.8 \mathrm{ng} / \mathrm{mL}$ in the controls, EGFR-negative patients, and EGFR-positive patients, respectively (Figure 1B). The median VEGF and MMP-9 values were significantly higher in patients with HCC, including EGFR-negative and -positive patients, than those in the controls $(P<0.01$, Figure 1). The difference in serum VEGF level between the EGFR-negative and -positive patients was nonsignificant $(P>0.05)$. However, the serum concentration of MMP-9 in the EGFR-positive patients with primary HCC was significantly higher than that of the EGFRnegative patients $(P<0.05)$. Serum VEGF and MMP-9 levels were significantly higher in patients with HCC than those in healthy controls. Mean serum VEGF and MMP-9 levels in the EGFR-negative and -positive patients were both twofold higher than those in the controls, respectively.

\section{Relationship Between Serum VEGF and MMP-9 Levels and Clinicopathological Characteristics in Patients with Primary $\mathrm{HCC}$}

The EGFR-negative and -positive patients with primary HCC were examined for the differences in VEGF and MMP-9 levels according to various clinicopathological characteristics (Table 2). As shown in Table 2, in the EGFR-negative and -positive patients, differences in serum VEGF or MMP-9 concentration by gender and age were nonsignificant $(P>0.05)$. Similarly, in the EGFR-negative or -positive patients with primary HCC, differences in serum VEGF level by stage, metastasis, and differentiation were nonsignificant $(P>0.05)$. However, for EGFR-positive patients with primary HCC, differences in stage, metastasis, and differentiation were significant $(P<0.05)$. Serum MMP-9 concentration was the highest at stage III in the EGFR-positive patients $(P<0.05)$, although the increase was not gradual in patients with stage I-IV primary HCC. In the EGFR-negative or positive patients, metastatic patients had lower MMP-9 levels than nonmetastatic patients, respectively. The differences in serum MMP-9 level between EGFR-positive patients with metastasis and EGFR-positive patients without metastasis were significant $(P<0.05)$ but not in EGFR-negative patients $(P>0.05)$. For EGFR-positive patients, serum MMP-9 level in recurrent patients was significantly higher than that in non-recurrent patients $(P<0.05)$. However, in the EGFR-negative and -positive patients, there were no conspicuous differences in the 
A

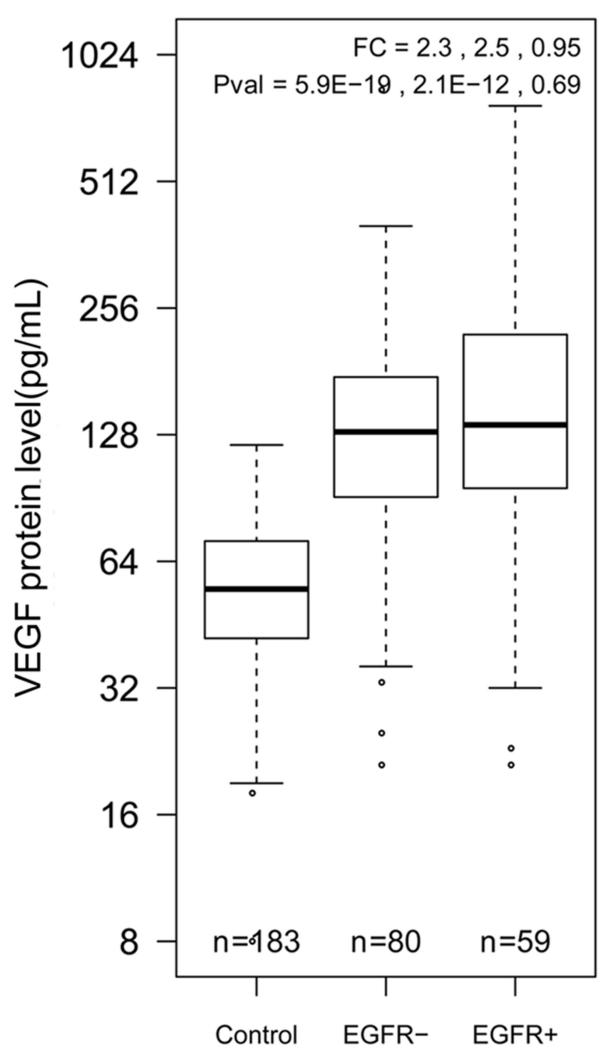

B

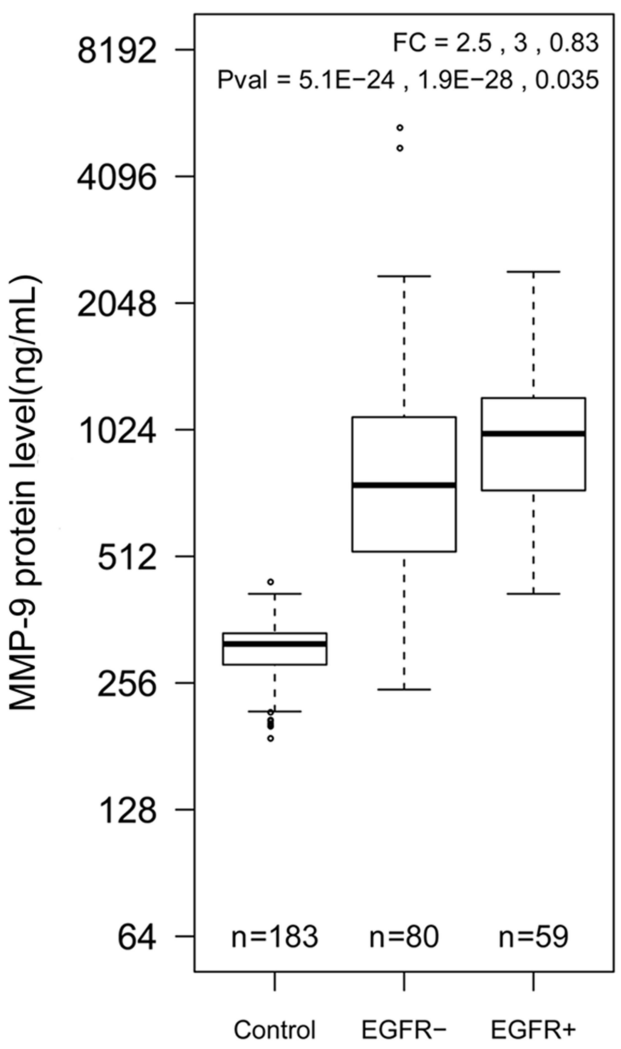

Figure I Boxplots of serum VEGF and MMP-9 in healthy controls, EGFR-negative patients, and EGFR-positive patients. (A) Serum VEGF concentration in three different groups. (B) Serum MMP-9 concentration in three different groups. Fold change and P-values are listed in the order of control vs EGFR-positive patients, and EGFR-negative patients vs EGFR-positive patients.

Abbreviations: EGFR-, EGFR-negative patients with HCC and EGRF+, EGFR-positive patients with HCC.

concentrations of VEGF or MMP-9 between HBsAg $(+)$ and HBsAg $(-)$ status, respectively $(P>0.05)$.

\section{Relevance of EGFR Between Serum VEGF and MMP-9 in Patients with HCC That Received Different Chemotherapeutic Drugs in TACE Therapy}

A total of 139 patients with HCC were treated with TACE therapy at three different intervals (ie, first, second, and third courses of post-treatment), who received different chemotherapeutic drugs. The mean decrease in serum VEGF level in recurrent patients treated with TACE therapy was almost equal to that in non-recurrent patients at the first and second courses of treatment (Figure 2A). However, the mean decrease in serum MMP-9 level in non-recurrent patients was smaller compared to that in recurrent patients at the first, second and third courses of treatment (Figure 2B). Compared with the EGFR-positive patients, the mean decrease in serum VEGF level in the EGFR-negative patients was greater at the first course of treatment, but smaller at the third course of treatment (Figure 2C). As shown in Figure 2D, serum MMP-9 level in the EGFR-negative patients had greater decrease than that in the EGFRpositive patients after the first, second and third courses of treatments, respectively. According to different chemotherapeutic drugs within TACE therapy, 139 patients with HCC were divided into three groups, namely, E, $\mathrm{P}$, and $\mathrm{O}$ groups. As shown in Figure 2E, compared with the $\mathrm{E}$ and $\mathrm{O}$ groups, the $\mathrm{P}$ group showed a greater mean decrease in serum VEGF level at the first, second and third courses of treatments. However, the degree of the decrease in MMP-9 tended to be consistent among three groups after the first, second and third courses of treatments (Figure 2F). Median serum VEGF concentrations at the first course of treatments were almost equal to that of pre-treatment in the EGFR-negative and positive patients from the E group, respectively (Figure 3A). Compared to the pre-treatment, serum VEGF levels began to decrease at the second and third courses of treatment in the EGFR-positive and -negative patients, although the 
Table 2 Relation of Serum VEGF and MMP9 to Clinicopathological Characteristics of 139 Patients with HCC (Concentration Unit: VEGF, Pg/mL; MMP-9, Ng/mL)

\begin{tabular}{|c|c|c|c|c|c|c|c|}
\hline Group & EGFR & & $\mathbf{N}$ & VEGF Mean (SD) & $P$ value & MMP-9 Mean (SD) & $P$ value \\
\hline \multirow[t]{4}{*}{ Gender } & + & Male & 49 & $176.0(\mid 44.5)$ & & $1020.4(443.4)$ & \\
\hline & & Female & 10 & $160.4(84.2)$ & 0.744 & $987.3(370.3)$ & 0.826 \\
\hline & - & Male & 64 & $157.9(\mid 22.2)$ & & I007.5(847.2) & \\
\hline & & Female & 16 & $136.6(63.8)$ & 0.505 & $665.8(343.7)$ & 0.119 \\
\hline \multirow[t]{6}{*}{ Age } & + & $<50 \mathrm{yr}$ & 14 & $175.0(94.1)$ & & 1089.6(5I5.9) & \\
\hline & & $50-70 y r$ & 43 & $178.0(\mid 48.6)$ & & $981.7(395.8)$ & \\
\hline & & $>70 \mathrm{yr}$ & 2 & $62.0(8.5)$ & 0.505 & $1202.0(666.1)$ & 0.596 \\
\hline & - & $<50 \mathrm{yr}$ & 22 & $130.9(8 \mid .0)$ & & III $0.0(929.1)$ & \\
\hline & & $50-70 y r$ & 47 & $165.8(130.2)$ & & $922.9(776.3)$ & \\
\hline & & $>70 \mathrm{yr}$ & 11 & |47.I(84.4) & 0.485 & $667.4(341.9)$ & 0.306 \\
\hline \multirow[t]{8}{*}{ Stage } & + & I & 4 & $125.0(86.3)$ & & $770.5(313.5)$ & \\
\hline & & II & 19 & $162.4(177.1)$ & & $908.1(398.9)$ & \\
\hline & & III & 25 & I70.I(90.2) & & $1215.6(449.9)$ & \\
\hline & & IV & 11 & $217.3(158.8)$ & 0.626 & $831.5(287.9)$ & 0.015 \\
\hline & - & I & 18 & $185.5(\mid 75.6)$ & & $682.5(403.6)$ & \\
\hline & & II & 40 & $151.3(92.0)$ & & $841.6(320.2)$ & \\
\hline & & III & 10 & 139.9(88.9) & & $\mid 273.5(|35| .0)$ & \\
\hline & & IV & 12 & $124.9(68.8)$ & 0.506 & $1370.9(1360.9)$ & 0.142 \\
\hline \multirow[t]{4}{*}{ Metastasis } & + & Yes & 13 & $181.5(160.7)$ & & $804.3(308.5)$ & \\
\hline & & No & 46 & $171.0(\mid 29.7)$ & 0.808 & I074.3(442.2) & 0.044 \\
\hline & - & Yes & 11 & $108.2(34.7)$ & & $715.5(294.3)$ & \\
\hline & & No & 69 & $160.9(119.4)$ & 0.152 & $974.9(831.2)$ & 0.311 \\
\hline \multirow[t]{4}{*}{ Recurrence } & + & Yes & 24 & I46.I(||7.1) & & II 57.5(522.0) & \\
\hline & & No & 35 & 192.I(|45.8) & 0.204 & $916.9(324.8)$ & 0.033 \\
\hline & - & Yes & 34 & $146.8(\mid 37.1)$ & & $1 \mathrm{II} 3.6(1 \mathrm{I} 10.0)$ & \\
\hline & & No & 46 & $158.7(92.5)$ & 0.644 & $810.3(366.1)$ & 0.087 \\
\hline \multirow[t]{4}{*}{$\mathrm{HBsAg}$} & + & + & 27 & $180.5(|| 4.8)$ & & I061.3(489.8) & \\
\hline & & - & 32 & $167.3(\mid 52.7)$ & 0.713 & $975.5(373.9)$ & 0.449 \\
\hline & - & + & 52 & $156.0(123.5)$ & & $874.9(742.0)$ & \\
\hline & & - & 28 & $149.1(92.1)$ & 0.797 & $1058.5(856.2)$ & 0.320 \\
\hline \multirow[t]{6}{*}{ Differentiation } & + & High & 4 & $125.0(86.3)$ & & $770.5(313.5)$ & \\
\hline & & Moderate & 38 & $176.0(139.0)$ & & $1119.3(46 I .1)$ & \\
\hline & & Low & 17 & $178.8(|4| .6)$ & 0.766 & $838.6(289.0)$ & 0.038 \\
\hline & - & High & 19 & $\mid 82.9(|7| .0)$ & & 699.1(398.8) & \\
\hline & & Moderate & 43 & $151.5(87.9)$ & & $950.7(705.6)$ & \\
\hline & & Low & 18 & |27.8(87.0) & 0.331 & II65.I(II55.3) & 0.194 \\
\hline
\end{tabular}

differences were non-significant $(P>0.05)$ (Figure 3A). In the E group, serum MMP-9 levels at the third course of treatment were the lowest in both the EGFR-positive and negative patients $(P<0.05)$. Additionally, serum MMP-9 concentration decreased from $760 \mathrm{ng} / \mathrm{mL}$ at the pretreatment to $350 \mathrm{ng} / \mathrm{mL}$ at the third course of treatment in the EGFR-negative patients from the E group. Serum MMP9 concentration increased in the EGFR-positive patients after the first course of treatment compared with that at the pretreatment. However, their serum MMP-9 levels gradually decreased after the second and third courses of treatments (Figure 3B). 

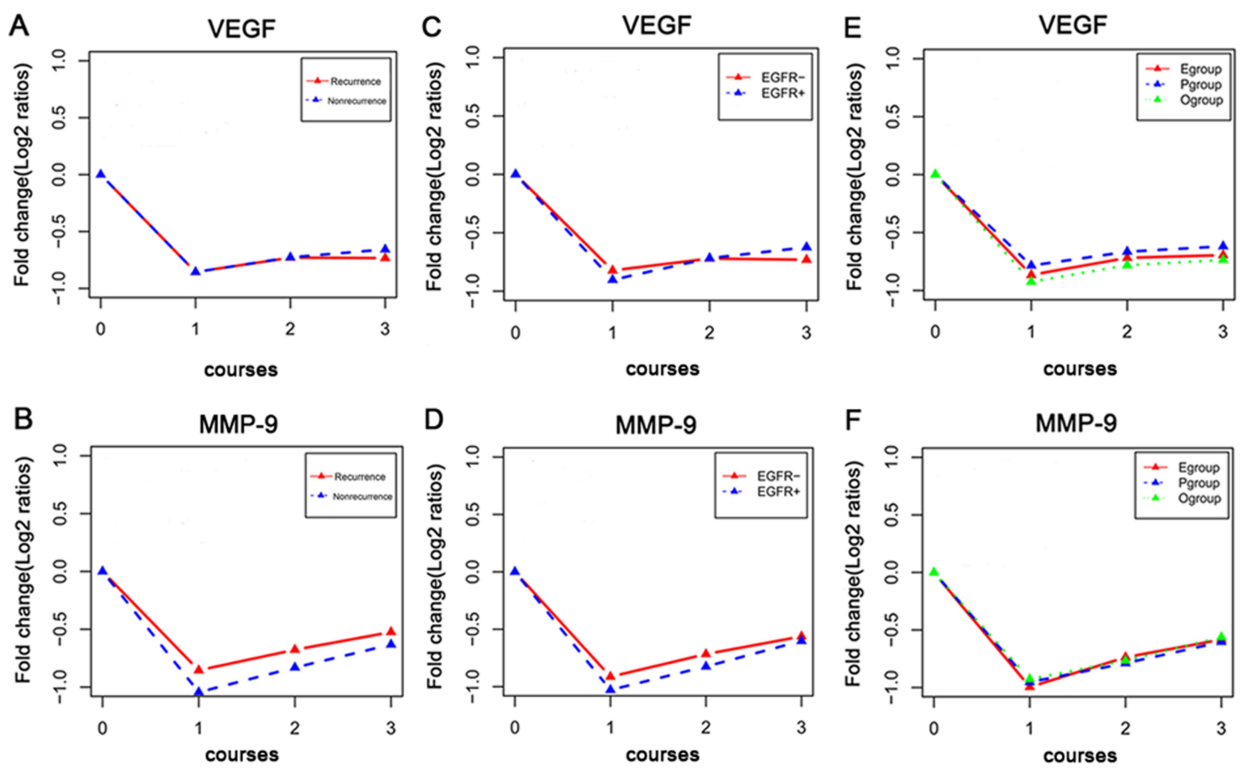

Figure 2 Changes in serum VEGF and MMP-9 levels in patients with HCC after TACE therapy. Mean changes in serum (A) VEGF and (B) MMP-9 levels between recurrent and non-recurrent patients after TACE therapy. Mean changes in serum (C) VEGF and (D) MMP-9 levels between EGFR-negative and -positive patients after TACE therapy. Mean changes in serum (E) VEGF and (F) MMP-9 levels in patients with HCC after TACE therapy with different chemotherapeutic drugs relative to the pre-treatment value. Abbreviations: EGFR-, EGFR-negative patients with HCC; EGRF+, EGFR-positive patients with HCC; E group, patients treated with adriamycin and its isomers regimen are included; $P$ group, patients treated with platinum regimen are included; and $O$ group, patients treated with other regimens are included.

E group

A

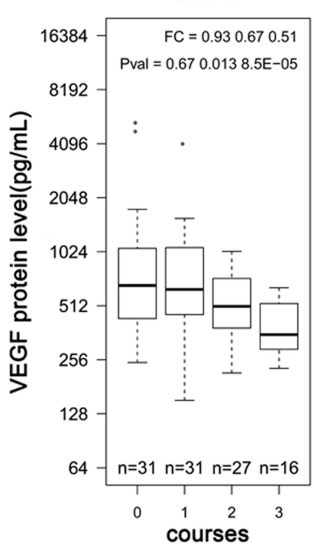

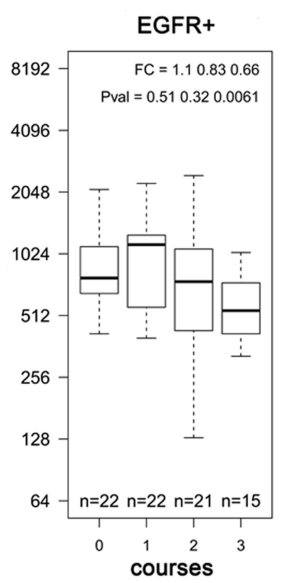

B

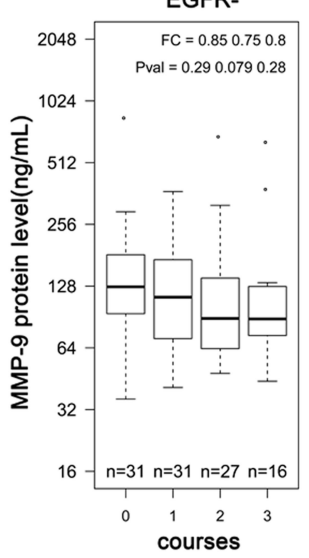

EGFR+

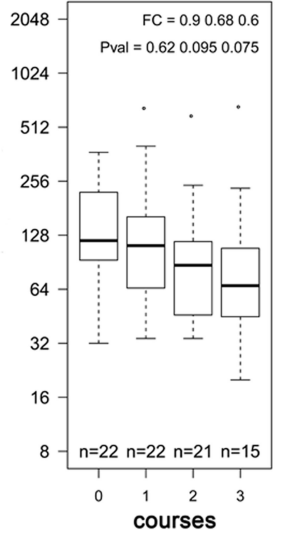

Figure 3 Boxplots of serum (A) VEGF and (B) MMP-9 levels in EGFR-negative and -positive patients after TACE therapy with chemotherapeutic drugs based on adriamycin and its isomers.

Abbreviations: EGFR-, EGFR-negative patients with HCC and EGRF+, EGFR-positive patients with HCC.

Compared with $155 \mathrm{pg} / \mathrm{mL}$ at the pre-treatment, serum VEGF concentration ranged from $105 \mathrm{pg} / \mathrm{mL}$ to $130 \mathrm{pg} / \mathrm{mL}$ within the first, second and third courses of treatments in the EGFR-positive patients after TACE therapy with platinum $(P>0.05)$. Serum VEGF concentration tended to decrease from $130 \mathrm{pg} / \mathrm{mL}$ at the pre-treatment to $70 \mathrm{pg} / \mathrm{mL}$ at the third course of treatment in the EGFR-negative patients subjected to TACE therapy with platinum $(P<0.05)$ (Figure 4A). Although the median MMP-9 concentration decreased from
$1050 \mathrm{ng} / \mathrm{mL}$ at the pre-treatment to $520 \mathrm{ng} / \mathrm{mL}$ at the third course of treatment in the EGFR-positive patients $(P<0.05)$, serum MMP-9 level after the first and second courses of treatments maintained a high level and approached the level obtained at the pre-treatment $(P>0.05)$. However, the MMP9 concentration gradually decreased from $800 \mathrm{ng} / \mathrm{mL}$ at the pre-treatment to $470 \mathrm{ng} / \mathrm{mL}$ at the third course of treatment in the EGFR-negative patients that received TACE therapy with platinum $(P<0.05)$ (Figure 4B). 


\section{$P$ group}

A

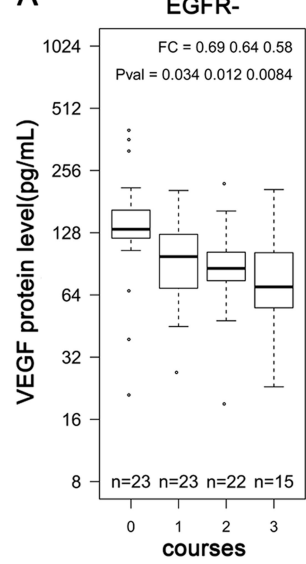

B

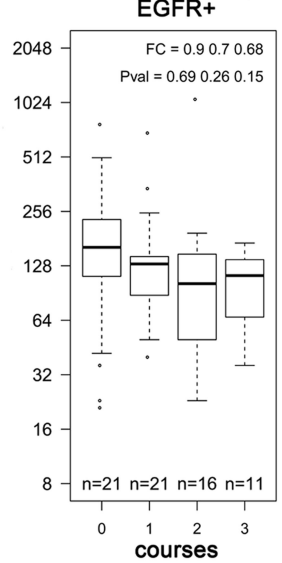

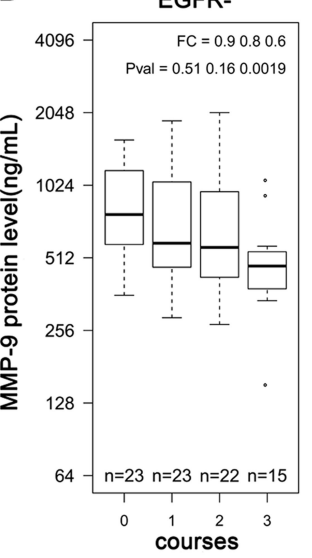

EGFR+

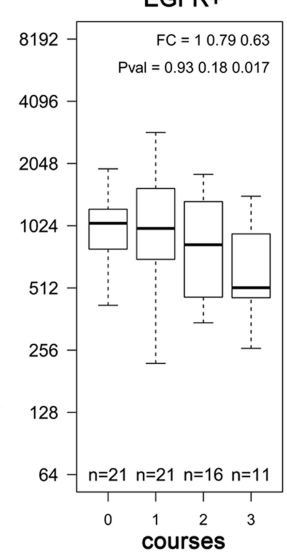

Figure 4 Boxplots of serum (A) VEGF and (B) MMP-9 levels in EGFR-negative and -positive patients after TACE therapy with chemotherapeutic drugs based on platinum. Abbreviations: EGFR-, EGFR-negative patients with HCC and EGRF+, EGFR-positive patients with HCC.

Serum VEGF level after the first course of treatment was approximate to that at the pre-treatment in both EGFR-positive and -negative patients in the $\mathrm{O}$ group, respectively. The slight decreases of serum VEGF levels were observed after the second and third courses of treatments in the EGFR-positive and -negative patients, respectively $(P>0.05)$ (Figure 5A). Serum MMP-9 level at the first course of treatment did not decrease in the EGFRnegative patients that received TACE therapy in the O group. However, serum MMP-9 levels were approximately $512 \mathrm{ng} / \mathrm{mL}$ at the second and third courses of treatments in the EGFR-negative patients $(P<0.05)$. The median values of MMP-9 at the first and second courses of treatments were approximately $1000 \mathrm{ng} / \mathrm{mL}$ which decreased slightly in the EGFR-positive patients that received TACE therapy in the $\mathrm{O}$ group $(P>0.05)$. However, MMP-9 concentration decreased to approximately $512 \mathrm{ng} / \mathrm{mL}$ at the third course of treatment in the EGFR-positive patients from the $\mathrm{O}$ group (Figure 5B).

The longitudinal change of serum VEGF and MMP-9 for each individual is plotted in Figure 6. The data indicate that VEGF and MMP-9 concentrations were continuously declined in most individuals at the first, second and third course of treatment among three groups. However, serum VEGF concentrations of few individuals increased quickly at the first, second and third courses of TACE therapy with different chemotherapeutic drugs in the EGFR-positive and -negative patients. Serum MMP-9 concentrations of

\section{O group}
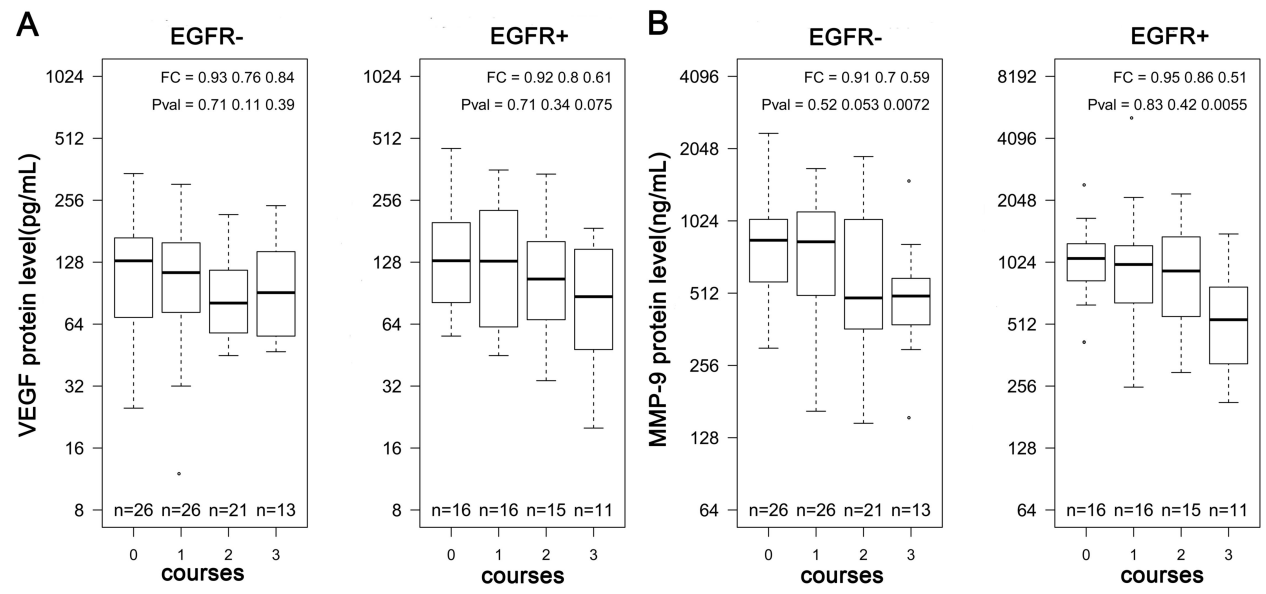

Figure 5 Boxplots of serum (A) VEGF and (B) MMP-9 levels in EGFR-negative and -positive patients after TACE therapy with chemotherapeutic drugs based on other drugs. Abbreviations: EGFR-, EGFR-negative patients with HCC and EGRF+, EGFR-positive patients with HCC. 

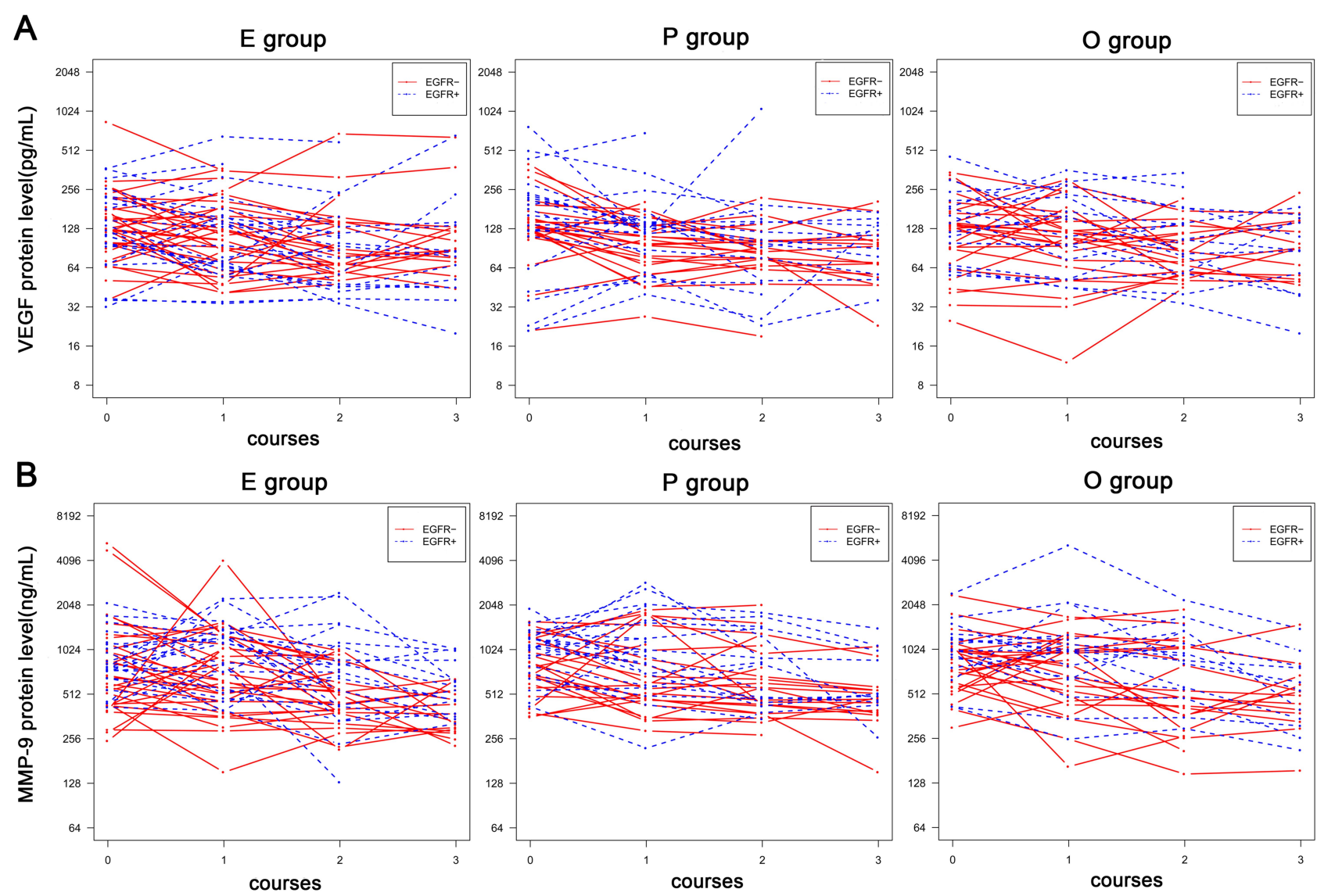

Figure 6 Longitudinal serum (A) VEGF and (B) MMP-9 in individual patients. Serum concentrations of VEGF and MMP-9 were plotted on the Y-axis and sampling time after therapy were plotted on the $X$-axis. Each dot represents a sample and different time points for the same patient is linked by a line.

Abbreviations: EGFR-, EGFR-negative patients with HCC; EGRF+, EGFR-positive patients with HCC; E group, patients treated with adriamycin and its isomers regimen are included; $\mathrm{P}$ group, patients treated with platinum regimen are included; and $\mathrm{O}$ group, patients treated with other regimens are included.

some individuals remained high at the second and third courses of treatments in EGFR-positive patients among the three groups.

\section{Discussion}

The incidence of HCC varies among regions, between genders, among ethnic groups, and among races and is closely associated with few risk factors, such as hepatitis $\mathrm{B}$ and hepatitis $\mathrm{C}$ infections. ${ }^{15}$ EGFR overexpression, which is correlated with tumor invasion, metastasis, and poor patient survival, occurs in $68 \%$ of patients with HCC. ${ }^{16}$ Although EGFR overexpression exists in most $\mathrm{HCC}$ cases, increase in EGFR protein expression does not correlate with $E G F R$ gene copy number. ${ }^{17}$ In the present study, 139 patients with primary HCC, composed of 113 males and 26 females, were selected. HCC is more common in men than in women. ${ }^{18} \mathrm{~A}$ total of $56.8 \%$ patients with primary HCC were positive with hepatitis B surface antigen in the present study. Approximately 50\% of new cases of HCC and deaths occurred in China owing to the high prevalence of chronic hepatitis B virus infection in this country. ${ }^{18}$ In the study, a total of $42.4 \%$ EGFRpositive patients were diagnosed by IHC staining among the investigated data, thereby suggesting that EGFRpositive expression was common in primary HCC. EGFR overexpression in males could be correlated to a subclass of HCC with polysomy of chromosome $7 .{ }^{19}$

High microvessel density was observed in patients with HCC having EGFR-positive tumor endothelial cells. ${ }^{2}$ MMP-9 is a member of the MMP family and plays an important role in tumor angiogenesis and VEGF bioavailability regulation. ${ }^{20}$ VEGF stimulates angiogenesis by providing nutrients and oxygen needed for metabolism, ${ }^{21}$ decreases the functions of the biological barrier, and regulates endothelial sprouting. ${ }^{22}$ VEGF or MMP-9 plays important roles in tumor angiogenesis and has clinical significance in the diagnosis, treatment, and prognosis of various cancers, including $\mathrm{HCC} .{ }^{23-26}$ EGFR inhibitor can decrease the tumorigenesis of LC cells by regulating the ERK-EGF/MMP-9 signaling pathway. ${ }^{27}$ Thus, EGFR may 
have important correlation with VEGF and MMP-9 in tumor progression. In the present study, serum VEGF and MMP-9 levels in patients with HCC were significantly higher than those in the healthy controls $(P<0.05)$. This finding is consistent with the findings of previous reports. $^{23,26}$ In particular, serum MMP-9 level was significantly high in the EGFR-positive patients than in the EGFR-negative patients $(P<0.05)$. However, the difference in serum VEGF level between the EGFR-negative and -positive patients was nonsignificant $(P>0.05)$. The differences in serum MMP-9 level by in stage, metastasis, and differentiation were significant in the EGFR-positive patients compared with the EGFR-negative patients $(P<0.05$, Table 2$)$. These results indicated a compact correlation between EGFR expression and serum MMP-9 level, rather than VEGF, in the EGFR-positive patients with primary HCC. A possible reason is that MMP-9 can activate EGFR in either tumorigenesis or neoplastic angiopoiesis. After partial hepatectomy, MMP-9 deficiency impairs liver regeneration by inhibiting and delaying EGFR activation. The expression levels of EGFR ligands, such as HB-EGF, are relatively low in MMP-9 knockout mice. ${ }^{28}$ VEGF stimulates endothelial cell proliferation by activating MMPs, thereby inducing tumor angiogenesis. ${ }^{29}$ MMP-9 is critical to tumor invasion and metastasis and considered a tumor angiogenic factor that is involved in the signaling systems of VEGF-VEGF receptors. ${ }^{30}$ Therefore, MMP-9 can be used as a potential biomarker to indicate tumor neovascularization, invasion, and metastasis and differentiate stage, metastasis, and differentiation in EGFR-positive patients with primary HCC. Furthermore, the EGFR system is suggested to contribute to immune tolerance and viral amplification after Hepatitis B Virus (HBV) infection. ${ }^{2}$ While HBV $x$-gene product has been proven to induce EGFR overexpression, HBV-encoded $\mathrm{x}$ protein indirectly downregulated EGFR expression in HCC cells. ${ }^{31,32}$ In the study, the levels of VEGF and MMP-9 were not affected by HBsAg $(+)$ or HBsAg (-) status in the EGFR-positive and negative patients $(P>0.05$, Table 2$)$. The possible reason is that there is no correlation between HBsAg status and tumor angiogenesis in HCC patients.

TACE is currently used as first-line therapy for intermediate-stage HCC (Barcelona Clinic LC [BCLC] stage B), although this stage comprises patients with a wide range of liver functions and tumors, which are variable in number and size. ${ }^{33}$ TACE is generally repeated for maximum tumor recession because only $50 \%-60 \%$ of patients with BCLC stage B benefit from TACE in clinical practice. $^{34}$ In the present study, the enrolled patients received TACE therapy with different chemotherapeutic drugs at three different intervals (the first, second, and third courses of treatments). Serum VEGF and MMP-9 levels decreased in the EGFR-positive and -negative patients with TACE therapy at the first, second and third courses of treatments (Figure 2C and D). Serum VEGF and MMP-9 levels showed slight decrease in the EGFRpositive and -negative patients at the first course of treatment among three groups, except serum VEGF level in the EGFR-negative patients from the $\mathrm{P}$ group $(P>0.05$, Figures 3-5). Serum VEGF level in the EGFR-positive patients among three groups slightly decreased at the first, second and third courses of treatments; however, the differences were not significant $(P>0.05$, Figure $3 \mathrm{~A}$, 4A and 5A). Serum MMP-9 level in the EGFR-positive patients among three groups showed mild decrease at the first and second courses of treatments; however, the decreases at the third course of treatment were significant $(P<0.05$, Figure 3B, 4B and 5B).

These results indicated that serum VEGF and MMP9 levels showed slight decrease in EGFR-positive patients at the second courses of treatments after TACE therapy with different chemotherapeutic drugs (Figures 3-5). Serum VEGF level significantly decreased at the second course of treatment in the EGFR-negative patients from the P group $(P<0.05$, Figure 4A), while serum MMP-9 level significantly decreased at the second course of treatment in the EGFR-negative patients from the E group $(P<$ 0.05, Figure 3B). Compared with EGFR-negative patients with primary HCC, EGFR-positive patients showed low sensitivity to TACE therapy with different chemotherapeutic drugs. The absence of curative treatment methods has encouraged extensive research on HCC for the development of new therapeutic strategies. ${ }^{35}$ Therefore, several researchers attempted to clarify whether two consecutive nonresponses are the optimal criteria for the introduction of a guideline to stop further TACE therapy for patients with HCC by evaluating their response to TACE. ${ }^{36}$ Evidence has confirmed the indispensable role for EGFR in $\mathrm{LC}^{2}{ }^{2}$ thereby indicating that targeted EGRF might be a useful marker and could represent a new therapeutic target for EGFR-positive patients with HCC. Tumorigenesis is linked with macrophage-mediated chronic inflammation and various signaling pathways, including EGFR pathway. However, paradoxical results have been found in the literature. The exact role of EGFR in HCC remains unclear, and EGFR-inhibitors 
showed disappointing clinical results. ${ }^{37}$ Nevertheless, the combination of TACE and antiangiogenic treatment, such as sorafenib, offers improved tumor control. ${ }^{38}$ According to a recent meta-analysis, the combination of TACE and sorafenib for unresectable HCC is superior in terms of time to progression. $^{39}$ Hence, EGFR-positive patients with HCC should strengthen treatment monitoring after TACC therapy, and new treatment strategies for patients who did not benefit from TACE therapy should be developed.

This study has several limitations. First, the uncertainty in the treatment process may lead to the potential deviation of the results. Given the changes in therapeutic strategies in the treatment process, a certain chemotherapeutic drug for few patients was chosen at the early stage of treatment, which was changed at a later treatment stage. Therefore, we divided into groups on the basis of chemotherapeutic drugs of first choice at the first treatment. Although no change in the uniqueness of patients was observed among the groups, and no other changes were performed on TACE technique during the treatment, possible bias resulting from the changes cannot be excluded. Second, the relatively small sample size may reduce the statistical power to detect differences between EGFR-positive and -negative patients within the first, second, and third courses after TACE therapy with three kinds of different chemotherapeutic drugs. After all, the number of patients, which participated in statistics according to treatments with different chemotherapeutic drugs, especially after the third course of treatment, was relatively small in the $\mathrm{E}, \mathrm{P}$, and $\mathrm{O}$ groups.

\section{Conclusion}

HCC is swiftly increasing in prevalence globally with a high mortality rate. EGFR overexpression commonly occurs in primary HCC, with $42.4 \%$ by IHC staining in this study. The serum MMP-9 concentration in the EGFR-positive patients with primary $\mathrm{HCC}$ was significantly higher than that of EGFR-negative patients $(P<0.05)$. Serum VEGF and MMP-9 levels gradually decreased in the EGFR-positive and negative patients within the first, second, and third courses of treatment after TACE therapy with different chemotherapeutic drugs, respectively $(P<0.05)$. However, the mean decrease in serum MMP-9 level in the EGFR-positive patients was smaller compared to that in EGFR-negative patients after TACE therapy. Compared to the pre-treatment, serum VEGF and MMP9 levels showed limited decrease at the first and second courses of treatment in EGFR-positive patients after TACE therapy with different chemotherapeutic drugs.
Serum VEGF or MMP-9 levels tended to decrease significantly in the EGFR-negative patients from $\mathrm{E}, \mathrm{P}$ and $\mathrm{O}$ groups at the second course of treatment compared with that at the pre-treatment. EGFR-positive patients with HCC should be subjected to strengthened treatment monitoring after TACC therapy, and new treatment strategies for patients who did not benefit from TACE therapy should be developed.

\section{Acknowledgments}

The present study was supported by the second batch of special funds for Xuzhou Medical Key Discipline Construction and Talent Cultivation in 2018 (2018XJ-2).

\section{Disclosure}

The authors report no conflicts of interest for this work.

\section{References}

1. Jemal A, Bray F, Center MM, Ferlay J, Ward E, Forman D. Global cancer statistics. CA Cancer J Clin. 2011;61(2):69-90. doi:10.3322/ caac. 20107

2. Komposch K, Sibilia M. EGFR signaling in liver diseases. Int J Mol Sci. 2015;17(1):pii: E30. doi:10.3390/ijms17010030

3. Shariff MI, Cox IJ, Gomaa AI, Khan SA, Gedroyc W, TaylorRobinson SD. Hepatocellular carcinoma: current trends in worldwide epidemiology, risk factors, diagnosis and therapeutics. Expert Rev Gastroenterol Hepatol. 2009;3(4):353-367. doi:10.1586/egh.09.35

4. Cheng AL, Kang YK, Chen Z, et al. Efficacy and safety of sorafenib in patients in the Asia-Pacific region with advanced hepatocellular carcinoma: a Phase III randomised, double-blind, placebo-controlled trial. Lancet Oncol. 2009;10(1):25-34. doi:10.1016/S1470-2045(08)70285-7

5. Ikeda S, Tsigelny IF, Skjevik ÅA, et al. Next-generation sequencing of circulating tumor DNA reveals frequent alterations in advanced hepatocellular carcinoma. Oncologist. 2018;23(5):586-593. doi:10.1634/theoncologist.2017-0479

6. Nikolova D, Chalovska V, Ivanova M, et al. Immunohistochemical expression of epidermal growth factor receptor in hepatocellular carcinoma. Pril (Makedon Akad Nauk Umet Odd Med Nauki). 2018;39(2-3):21-28. doi:10.2478/prilozi-2018-0038

7. Blobel CP. ADAMs: key components in EGFR signalling and development. Nat Rev Mol Cell Biol. 2005;6(1):32-43. doi:10.1038/ nrm1548

8. Ohtsu H, Dempsey PJ, Eguchi S. ADAMs as mediators of EGF receptor transactivation by G protein-coupled receptors. Am J Physiol Cell Physiol. 2006;291(1):C1-10. doi:10.1152/ajpcell.00620.2005

9. Ciardiello F, Tortora G. EGFR antagonists in cancer treatment. $N$ Engl J Med. 2008;358(11):1160-1174. doi:10.1056/NEJMra0707704

10. Voelkel NF, Vandivier RW, Tuder RM. Vascular endothelial growth factor in the lung. Am J Physiol Lung Cell Mol Physiol. 2006;290(2): L209-21. doi:10.1152/ajplung.00185.2005

11. Peschos D, Damala C, Stefanou D, et al. Expression of matrix metalloproteinase-9 (gelatinase B) in benign, premalignant and malignant laryngeal lesions. Histol Histopathol. 2006;21(6):603-8.

12. Chang SH, Kanasaki K, Gocheva V, et al. VEGF-A induces angiogenesis by perturbing the cathepsin-cysteine protease inhibitor balance in venules, causing basement membrane degradation and mother vessel formation. Cancer Res. 2009;69(10):4537-4544. doi:10.1158/ 0008-5472.CAN-08-4539 
13. Qiao Q, Zhang J, Wang W, Li Q. Over expression of transforming growth factor and epidermal growth factor receptor in human hepatic cirrhosis tissues. Hepatogastroenterology. 2008;55(81):169-172.

14. $\mathrm{Ma} \mathrm{R}, \mathrm{Xu} \mathrm{H}, \mathrm{Wu}$ J, et al. Identification of serum proteins and multivariate models for diagnosis and therapeutic monitoring of lung cancer. Oncotarget. 2017;8(12):18901-18913. doi:10.18632/oncotarget.14782

15. El-Serag HB. Hepatocellular carcinoma. N Engl J Med. 2011;365 (12):1118-1127. doi:10.1056/NEJMra1001683.

16. Ito Y, Takeda T, Sakon M, et al. Expression and clinical significance of ErbB receptor family in hepatocellular carcinoma. Br J Cancer. 2001;84(10):1377-1383. doi:10.1054/bjoc.2000.1580

17. Buckley AF, Burgart LJ, Sahai V, Kakar S. Epidermal growth factor receptor expression and gene copy number in conventional hepatocellular carcinoma. Am J Clin Pathol. 2008;129(2):245-251. doi:10.1309/WF10QAAED3PP93BH

18. Torre LA, Bray F, Siegel RL, Ferlay J, Lortet-Tieulent J, Jemal A. Global cancer statistics, 2012. CA Cancer J Clin. 2015;65(2):87-108. doi:10.3322/caac. 21262

19. Keng VW, Sia D, Sarver AL, et al. Sex bias occurrence of hepatocellular carcinoma in poly7 molecular subclass is associated with EGFR. Hepatology. 2013;57(1):120-130. doi:10.1002/hep.26004

20. Kessenbrock K, Plaks V, Werb Z. Matrix metalloproteinases: regulators of the tumor microenvironment. Cell. 2010;141(1):52-67. doi:10.1016/j.cell.2010.03.015

21. Bergers G, Benjamin LE. Tumorigenesis and the angiogenic switch. Nat Rev Cancer. 2003;3(6):401-410. doi:10.1038/nrc1093

22. Auyeung KK, Ko JK. Angiogenesis and oxidative stress in metastatic tumor progression: pathogenesis and novel therapeutic approach of colon cancer. Curr Pharm Des. 2017;23(27):3952-3961. doi:10.2174/1381612823666170228124105

23. Baitello ME, Tenani GD, Ferreira RF, et al. VEGF polymorphisms related to higher serum levels of protein identify patients with hepatocellular carcinoma. Can J Gastroenterol Hepatol. 2016;2016:9607054. doi:10.1155/2016/9607054

24. Lorente L. New prognostic biomarkers of mortality in patients undergoing liver transplantation for hepatocellular carcinoma. World J Gastroenterol. 2018;24(37):4230-4242. doi:10.3748/wjg.v24.i37.4230

25. Elewa MA, Al-Gayyar MM, Schaalan MF, et al. Hepatoprotective and anti-tumor effects of targeting MMP-9 in hepatocellular carcinoma and its relation to vascular invasion markers. Clin Exp Metastasis. 2015;32(5):479-493. doi:10.1007/s10585-015-9721-6

26. Lempinen M, Lyytinen I, Nordin A, et al. Prognostic value of serum MMP-8, -9 and TIMP-1 in patients with hepatocellular carcinoma. Ann Med. 2013;45(7):482-487. doi:10.3109/07853890.2013.823779
27. Chen Y, Chen X, Ding X, Wang Y. Afatinib, an EGFR inhibitor, decreases EMT and tumorigenesis of Huh-7 cells by regulating the ERK-VEGF/MMP-9 signaling pathway. Mol Med Rep. 2019;20 (4):3317-3325. doi:10.3892/mmr.2019.10562

28. Zhou B, Fan Y, Rao J, et al. Matrix Metalloproteinases-9 deficiency impairs liver regeneration through epidermal growth factor receptor signaling in partial hepatectomy mice. J Surg Res. 2015;197 (1):201-209. doi:10.1016/j.jss.2015.03.081

29. Hiratsuka S. Vasculogenesis, angiogenesis and special features of tumor blood vessels. Front Biosci. 2011;16:1413-1427. doi:10.2741/3796

30. Hiratsuka S, Nakamura K, Iwai S, et al. MMP-9 induction by vascular endothelial growth factor receptor-1 is involved in lung-specific metastasis. Cancer Cell. 2002;2(4):289-300. doi:10.1016/S15356108(02)00153-8

31. Menzo S, Clementi M, Alfani E, et al. Trans-activation of epidermal growth factor receptor gene by the hepatitis $\mathrm{B}$ virus $\mathrm{X}$-gene product. Virology. 1993;196(2):878-882. doi:10.1006/viro.1993.1550

32. Chen YJ, Chien PH, Chen WS, et al. Hepatitis B virus-encoded $\mathrm{x}$ protein downregulates EGFR expression via inducing microRNA-7 in hepatocellular carcinoma cells. Evid Based Complement Altern Med. 2013;2013:682380.

33. Marrero JA, Kulik LM, Sirlin CB, et al. Diagnosis, staging, and management of hepatocellular carcinoma: 2018 practice guidance by the American Association for the Study of Liver Diseases. Hepatology. 2018;68(2):723-750. doi:10.1002/hep.29913

34. Radu P, Dufour JF. Changing TACTICS in intermediate HCC: TACE plus sorafenib. Gut. 2020;pii: gutjnl-2020-320692.

35. Jindal A, Thadi A, Shailubhai K. Hepatocellular carcinoma: etiology and current and future drugs. J Clin Exp Hepatol. 2019;9(2):221-232. doi:10.1016/j.jceh.2019.01.004

36. Choi J, Lee D, Shim JH, et al. Evaluation of transarterial chemoembolization refractoriness in patients with hepatocellular carcinoma. PLoS One. 2020;15(3):e0229696. doi:10.1371/journal.pone.0229696

37. Lanaya H, Natarajan A, Komposch K, et al. EGFR has a tumor-promoting role in liver macrophages during hepatocellular carcinoma formation. Nat Cell Biol. 2014;16(10):972-977. doi:10.1038/ncb3031

38. Dufour JF, Johnson P. Liver cancer: from molecular pathogenesis to new therapies: summary of the EASL single topic conference. J Hepatol. 2010;52(2):296-304. doi:10.1016/j.jhep.2009.11.010

39. Li L, Zhao W, Wang M, et al. Transarterial chemoembolization plus sorafenib for the management of unresectable hepatocellular carcinoma: a systematic review and meta-analysis. BMC Gastroenterol. 2018;18(1):138. doi:10.1186/s12876-018-0849-0
OncoTargets and Therapy

\section{Publish your work in this journal}

OncoTargets and Therapy is an international, peer-reviewed, open access journal focusing on the pathological basis of all cancers, potential targets for therapy and treatment protocols employed to improve the management of cancer patients. The journal also focuses on the impact of management programs and new therapeutic

Submit your manuscript here: https://www.dovepress.com/oncotargets-and-therapy-journal agents and protocols on patient perspectives such as quality of life, adherence and satisfaction. The manuscript management system is completely online and includes a very quick and fair peer-review system, which is all easy to use. Visit http://www.dovepress.com/ testimonials.php to read real quotes from published authors. 